\title{
OVERGROWTH OF THE NEURAL TUBE IN YOUNG HUMAN EMBRYOS
}

BRADLEY M. PATTEN

Department of Anatomy, University of Michigan Medical School, Ann Arbor

ELEVEN FIGURES

In the routine examination of the serial sections of embryos newly added to the University of Michigan collection, a curious distortion of the central nervous system has attracted attention by its recurrence. With variations as to detail, the essential finding was what we began to call for lack of any better designation "overgrowth of the neural tube." The condition was most striking when seen in the developing brain. It appeared as if the neural tube epithelium had started to grow wildly so that it became folded, and refolded on itself, as it was crowded into a cranial space fairly normal in size and shape. When this condition was first noticed, it was dismissed with the mental comment that the preservation of the embryo had proved to be far worse than we expected when it was examined and photographed prior to sectioning. I subsequently learned by talking with Dr. Streeter, shortly before his death, that he had seen such conditions and had not paid much attention to them because he, too, had regarded them as the result of poor fixation. This conclusion was natural enough if one happened to see first a specimen in which the fixation as a whole was very poor. A section through the brain of such an embryo is illustrated in figure 1. Everyone accustomed to dealing with human embryological material has learned to expect a disappointingly large proportion of the embryos handled to be in poor histological condition. Even when they are promptly fixed after a spon- 
taneous abortion, most of them have suffered considerable deterioration by reason of having died in utero - often as much as several days - before they were finally expelled. Under such circumstances the tendency is to dismiss, quite casually, any material not satisfactorily preserved. I feel confident that this condition of neural tube overgrowth with which we are concerned has been repeatedly seen by other workers and passed over with little consideration, just as we overlooked the early instances of it that we encountered.

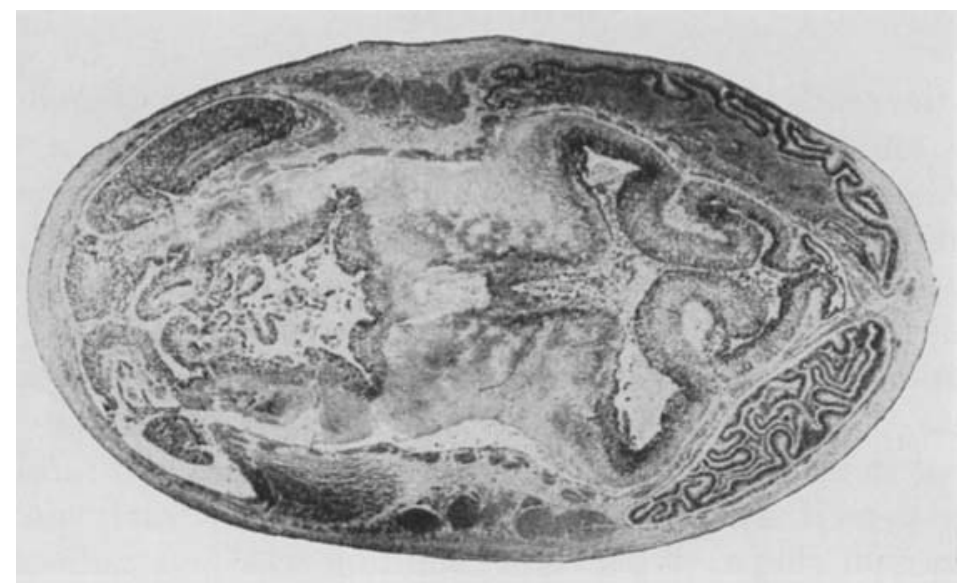

Fig. 1 Photomierograph of a section through the brain of a human embryo of $21 \mathrm{~mm}$ C-R (Univ. of Micl. Coll. EH 507). The external appearance of the specimen (see fig. 10, A) showed somewhat poor molding of the liead but scarcely sufficient distortion to lead one to anticipate anything like the extensive overgrowth of the neural tube that not even the poor fixation should cause one to overlook.

The first thing that led to the more critical rescrutinizing of such material was the finding of a series in which, along with conspicuous folding of the neural tube, the preservation of other structures in the body was reasonably good. If, for example, one looked only at the brain of the embryo illustrated in figure 2, it would be easy to dismiss the picture as meaning no more than poor fixation. If, however, one compares the condition of other organs as, for example, the heart, their 
structure is sufficiently well preserved to make one suspicious that something fundamentally different had occurred in the two areas. It is obvious from such things as the loosening of the superficial ectoderm from the underlying mesenchyme that preservation is far from perfect. Nevertheless, the thickened ectoderm of the nasal placodes is clearly recognizable. Another significant point is that the degenerative changes are most marked in the neural epithelium which has

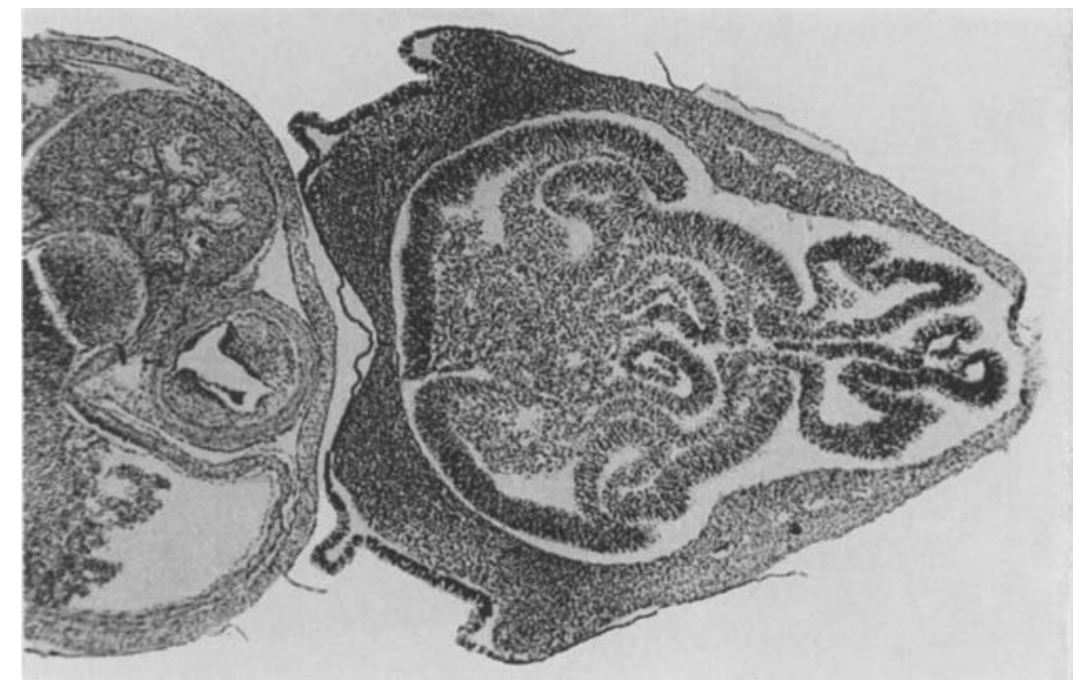

Fig. 2. Photomicrograph showing overgrowth in the forebrain of a human embryo of $9 \mathrm{~mm}$ (Univ. of Mich. Coll. EH 35). Note also the good preservation of structures in the cardiac region. The external appearance of this specimen before sectioning (fig. $7, B$ ) was surprisingly normal.

been most deeply infolded. This suggests that its deterioration began when it was folded in, away from the vascular mesenchyme which is normally adjacent to it. Certainly the more nearly normal appearance of the parts of the neural tube still in close relation to the surrounding mesenchyme and blood vessels is striking. These areas show, for the most part, the mediocre preservation that might be expected in the light of conditions in other parts of the body. The conclusion seems unescapable that there was an abnormal growth 
process going on in the neural tube before the intra-uterine death of the embryo. As happens in an expanding neoplasm, the parts of the rapidly growing tissue carried away from the normal source of their blood supply probably already had begun to degenerate before the death of the embryo. Following its death one suspects that the embryo remained in the uterus long enough to account for the mediocre fixation of all its tissues. Of course this would entail additional deterioration of the neural epithelium in the central areas already affected before its death.

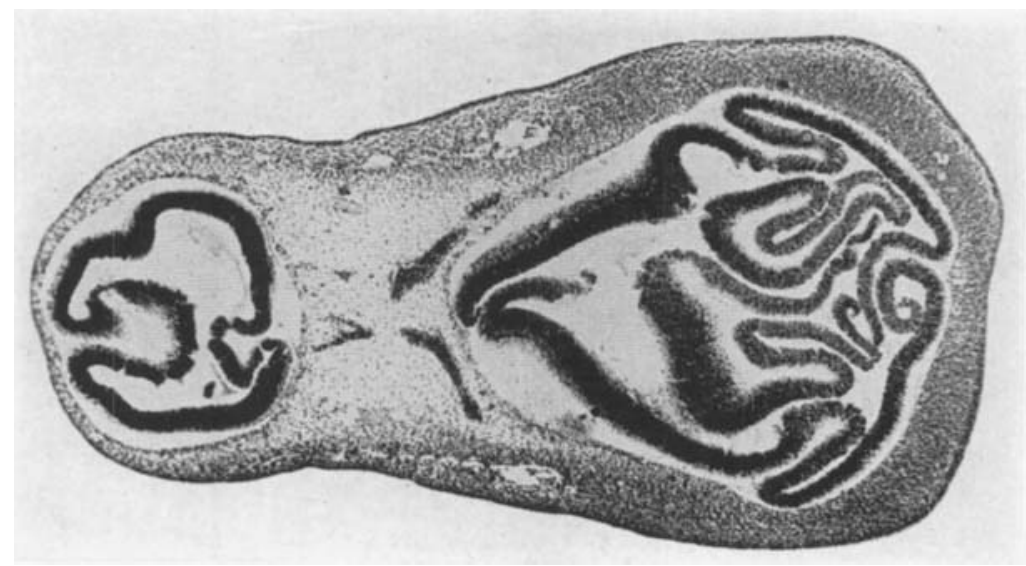

Fig. 3 Photomicrograph of a section showing extensive overgrowth of the neural epithelium of the fore- and midbrain in a human embryo of $7 \mathrm{~mm}$ (Unir. of Mich. Coll. EH 10). As in the previous case the external appearance of the embryo was essentially normal (see fig. $7, \mathrm{~A}$ ).

That this line of reasoning was probably correct seemed indicated by the conditions seen in other series that were restudied as interest in this condition developed. Take, for example, the picture presented by a $7 \mathrm{~mm}$ embryo in which the fixation was obviously quite good (fig. 3). The folding of the walls of the neural tube in both midbrain and forebrain is extraordinarily extensive. Only small portions of the most deeply infolded areas show any degenerative changes. Obviously this specimen as compared with that illustrated in figure 2 must have been fixed much more promptly after its 
death, but equally clearly the underlying disturbance must have been the same.

Of similar significance is the condition of the brain in a $10 \mathrm{~mm}$ embryo ( $\mathrm{EH} \mathrm{56).} \mathrm{In} \mathrm{this} \mathrm{case} \mathrm{the} \mathrm{folding} \mathrm{is} \mathrm{less}$ extensive (fig. 4). Either the severity of the involvement was less, or the process was caught at an early stage. The condition seen in the diencephalic region would nevertheless seem to be essentially similar to the other cases illustrated.

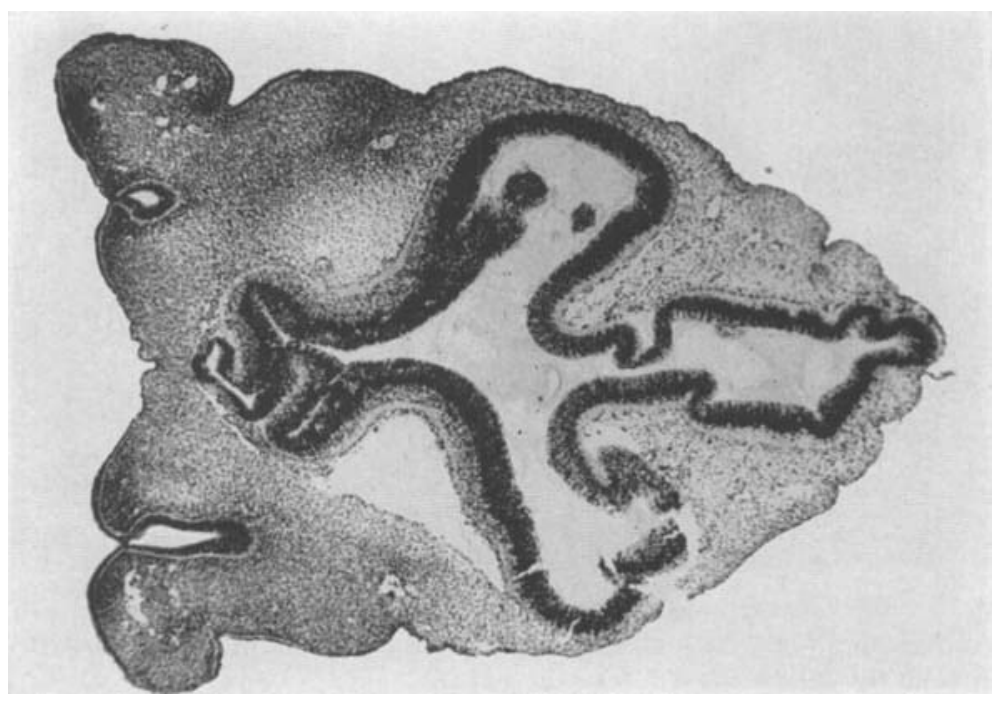

Fig. 4 Photomicrograph of a section showing overgrowth involving the diencephalic region of a human embryo of $10 \mathrm{~mm}$ (Univ. of Mich. Coll. FH 56).

Finally the bit of evidence that seemed definitely to settle the question of poor fixation was furnished by two pig embryos showing similar overgrowth. Since these specimens had been obtained from a slaughter house and fixed immediately, there was no possibility of postmortem degeneration being involved. The sections of these embryos speak for themselves (figs. 5, 6).

One of the most surprising things about the embrvos showing this overgrowth of their developing brain is that their external configuration may give almost no hint as to 
the radical internal disturbance. Figure 7 shows the external appearance of the embryos from which the sections shown in figures 2 and 3 were made. Other than to note some lack of clean-cut contours, one would be likely to regard the specimens as reasonably normal. Certainly one would be
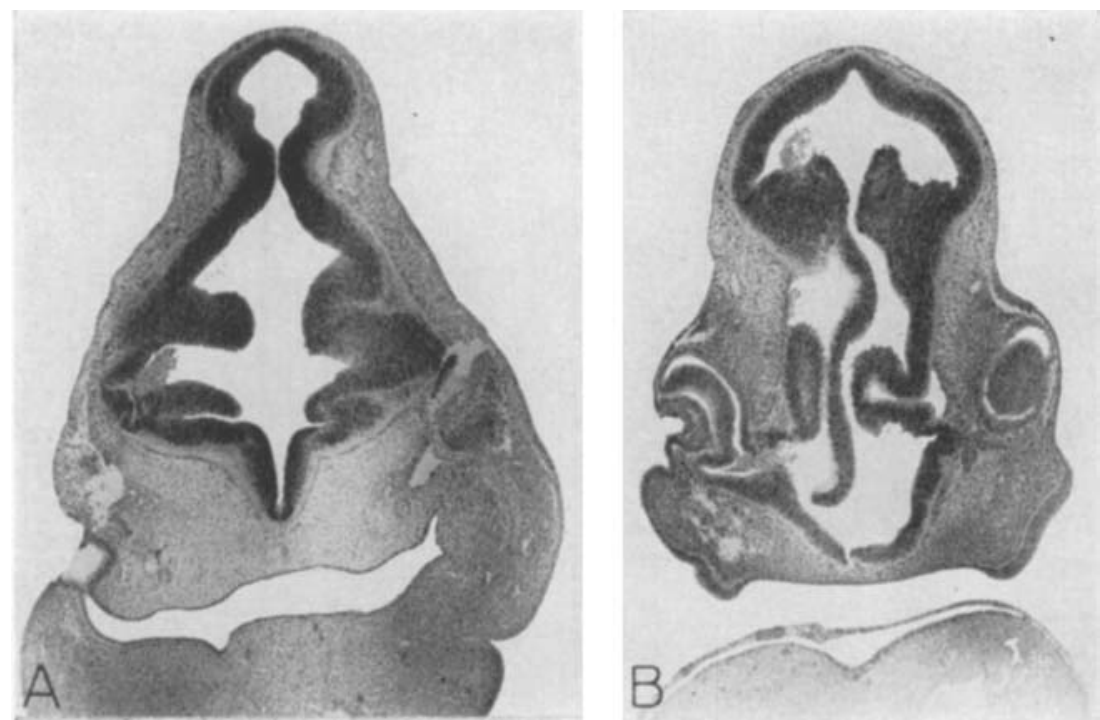

Fig. 5 Photomicrographs showing overgrowth involving the brain of a $5 \mathrm{~mm}$ pig embryo obtained from a slaughter house and fixed immediately. A and $B$ were taken from the same series a few sections apart.

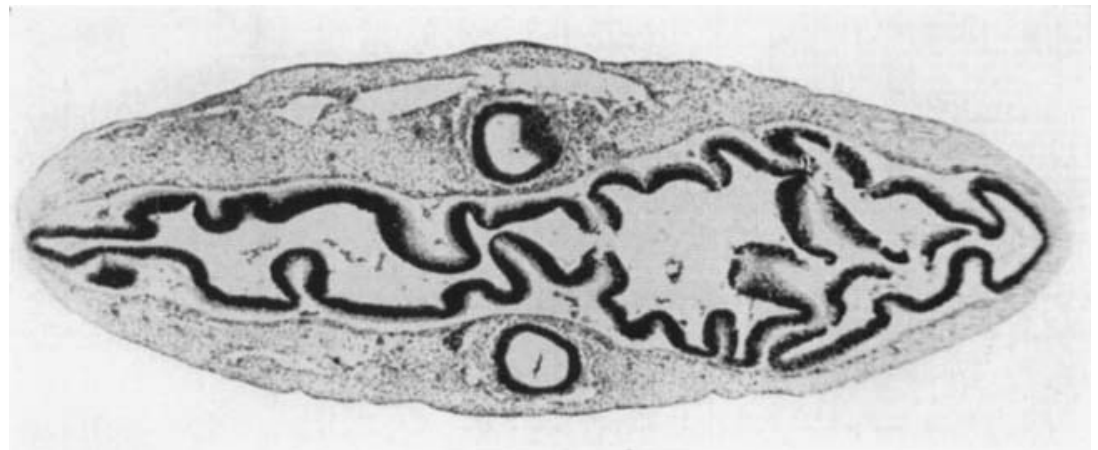

Fig. 6 Photomierograph showing strikingly overgrown neural epithelium in brain of another $5 \mathrm{~mm}$ pig embryo, also freshly fixed. 
totally unprepared for the marked abnormality exhibited when the serial sections were studied.

Although the external appearance of embryos with neural tube overgrowth may be surprisingly close to normal, this is not always the case. As one might expect the brain walls sometimes break through the thin overlying tissues (fig. 8). It is not necessarily the embryos with the most dramatic overgrowth in which the surface breakthrough occurs, as
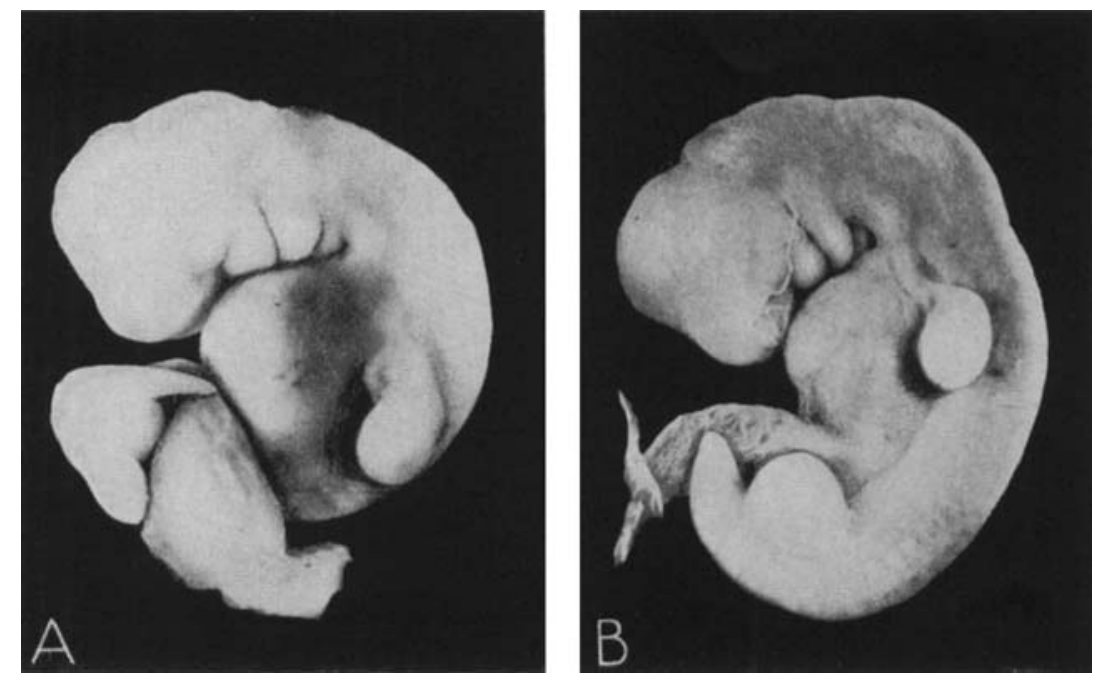

Fig. 7 Photographs of two fairly normal appearing embryos both of which showed extensive overgrowth of the brain when sectioned.

A. An embryo of $7 \mathrm{~mm}$ (Univ. of Mich. Coll. EH 10). See figure 3 for photomicrograph of typical section.

B. An. embryo of $9 \mathrm{~mm}$ (Univ. of Mich. Coll. EH 35). See figure 2 for photomicrograph of section.

is well indicated by the sections of the brain of the embryo here illustrated (cf. figs. 8 and 9).

In the light of the foregoing cases, it is apparent that external appearance is unreliable as a basis for assessing the existence or the extent of this particular anomaly of the developing neural tube. There are, however, certain types of cephalic configuration that we are beginning to look at with suspicion. Embryos in which the head is rather globular 
without external evidence of the regional divisions of the brain characteristic of normal embryos of corresponding size (fig. 10, A) often showed neural tube overgrowth. Also, a rather surprising number of embryos with heads of the tall ill-molded type that might be roughly characterized as conical (fig. 10, B) seem to show some degree of this type of disturbance. The
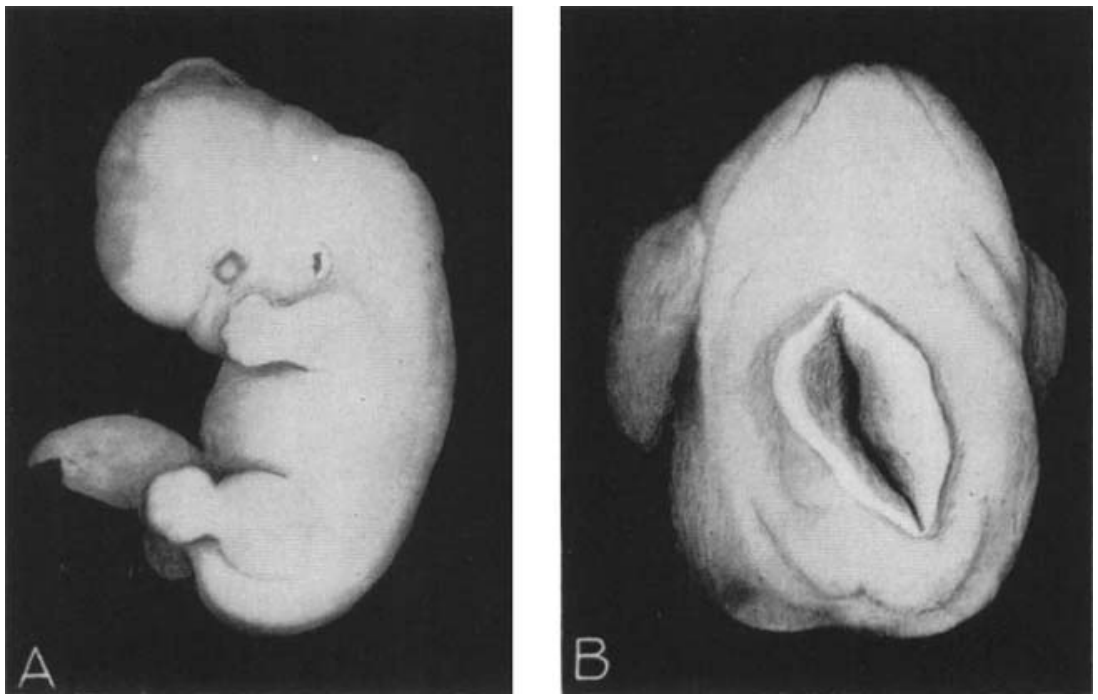

Fig. 8 External views of a $15 \mathrm{~mm}$ human embryo (Univ. of Mich. Coll. EH 227 ) showing the breaking through to the surface of an overgrown brain. A 1 aceident to the original negatives after the embryo was sectioned forced the making of the illustration from rather poor prints which had to be retouched to get sufficient contrast for reproduction.

A. Lateral view. B. Looking into the open defect on the top of the head.

numbers of specimens so far studied does not justify stating this as any more than a suggestive situation calling for further study.

Because they exemplify the condition so vividly, most of the illustrations presented so far have been based on sections through the brain. Although the brain appears to be involved more frequently, overgrowth is by no means limited to this part of the nervous system. A $12 \frac{1}{2} \mathrm{~mm}$ embryo which showed marked overgrowth in the brain (fig. 11, A) also showed a 
similar condition in the spinal cord (fig. 11, B), and in the retina (fig. 11, C). It is particularly striking that in the retina the central layer alone is involved, with the pigment layer showing its usual perfectly rounded contours. Nor is
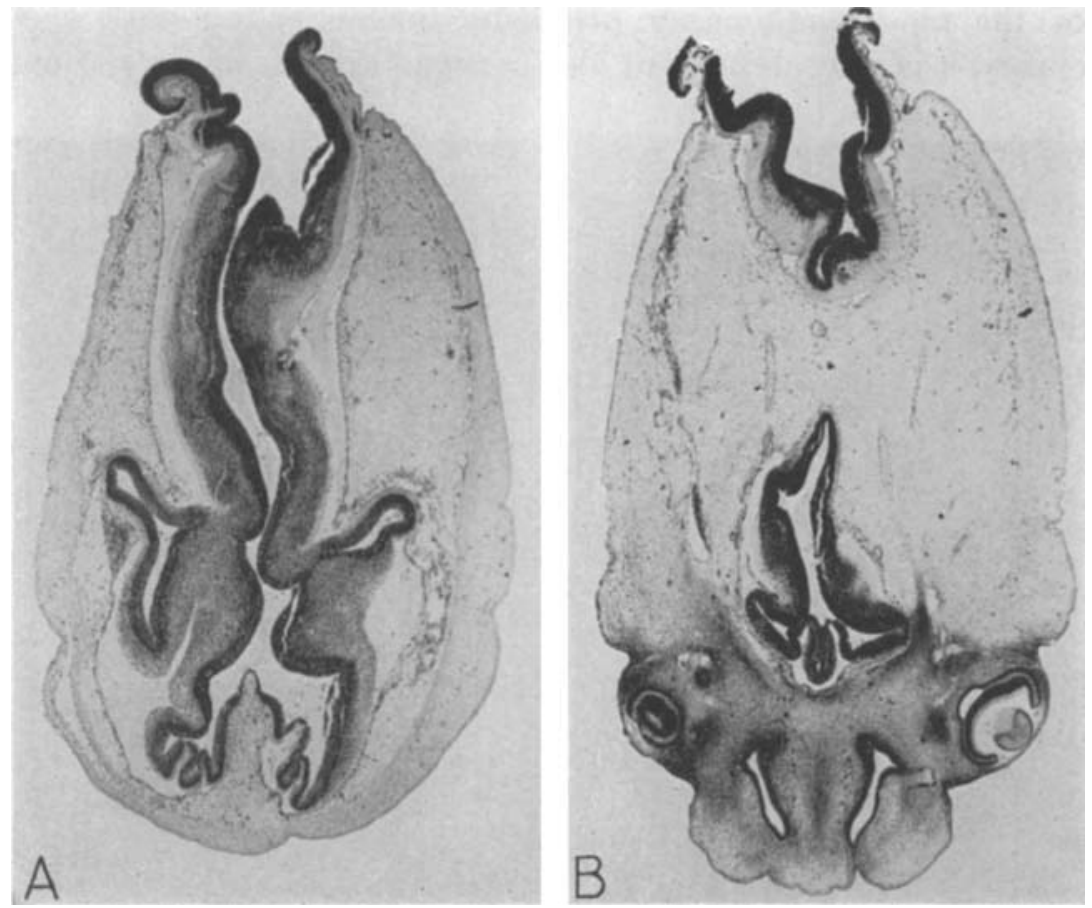

Fig. Photomicrographs of two sections through the head of the $15 \mathrm{~mm}$ embryo the external configuration of which was shown in figure 8 .

this condition of epithelial overgrowth entirely limited to the nervous system. In one of the more recently acquired embryos showing brain overgrowth there was a tendency though much less marked - for the gastric epithelium also to show some redundancy.

\section{DISCUSSTON}

The dramatic extensiveness of some of the cases of overgrowth of the walls of the young neural tube which have been here presented tempt one to indulge in interpretations which 
could readily slip over into mere speculation. It seems wiser at this time not to attempt to go beyond establishing the condition as a real and highly characteristic type of disturbance of development that has not been heretofore recognized, and that cannot be dismissed as the result of poor fixation. One of the most challenging problems in connection with such embryos is why defects of the nervous system of a type one
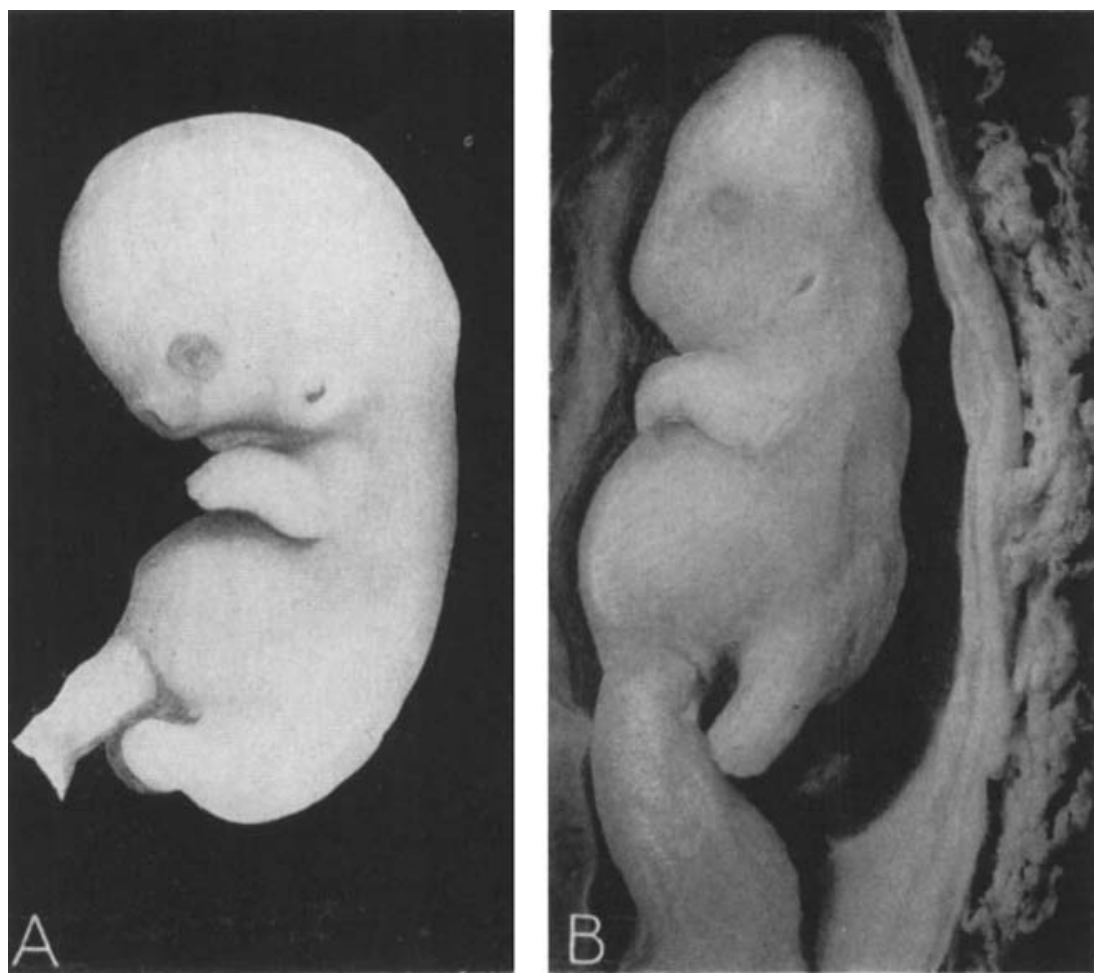

Fig. 10 Two embryos showing the somewhat distorted external configuration that sometimes accompanies neural tube overgrowth.

A. Human embryo of $21 \mathrm{~mm} \mathrm{C-R} \mathrm{(Univ.} \mathrm{of} \mathrm{Mich.} \mathrm{Coll.} \mathrm{FH} \mathrm{507).} \mathrm{Note} \mathrm{the}$ globular shape of the head without the clean-cut external configuration that normally gives indication of the regional divisions of the brain. For photomicrograph of a section through the head of the embryo see figure 1 .

B. Human embryo $20 \mathrm{~mm}$ C-R (Univ, of Mich. Coll. EH 162) showing ill-molded caudal region and a high conical head. The serial sections from this specimen showed overgrowth of the brain as extensive as that illustrated in figure 1 . 
could reasonably interpret as having originated from early overgrowth of the neural tube are not seen in the later stages of pregnancy or at birth. Are they changed by secondary degeneration into extensive defects that do not betray their origin, or are they all eliminated in the early stages of development? If they are weeded out, what causes the death
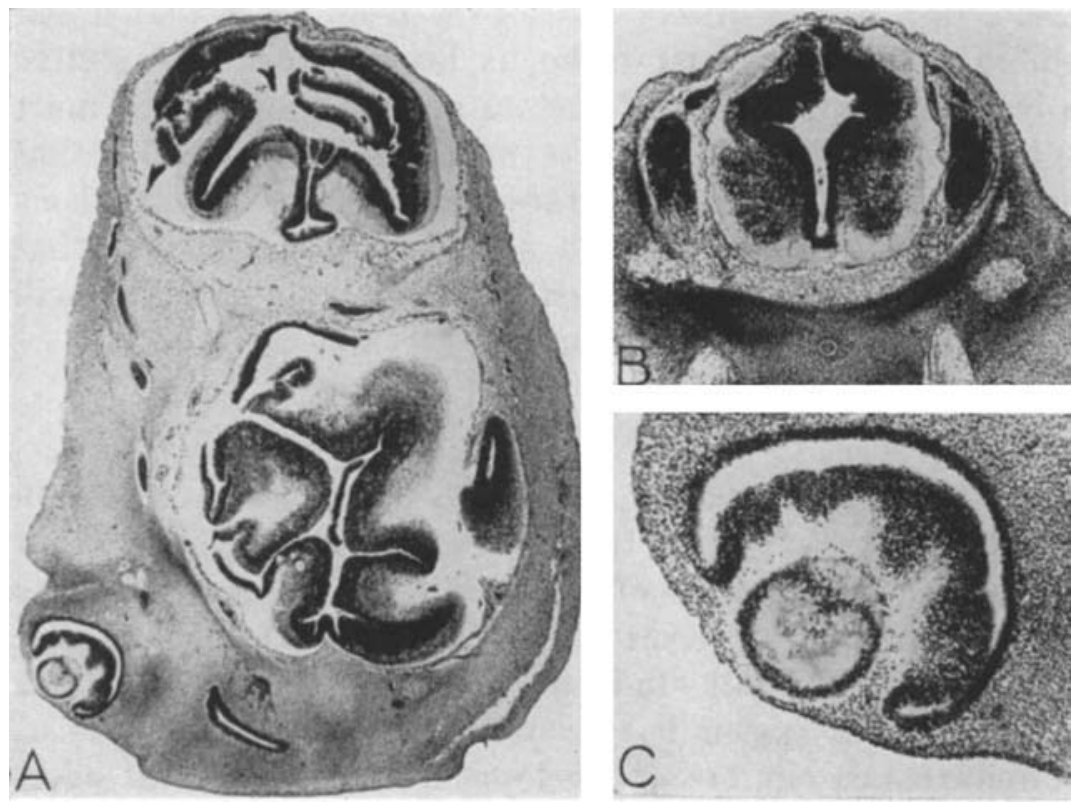

Fig. 11 Sections from various regions of a human embryo of $12.5 \mathrm{~mm}$ (Univ. of Mich. Coll. EH 16) showing overgrowth of neural epithelium.

A. Mesencephalon, diencephalon, and one of the eyes.

B. Spinal cord in thoracic region.

C. Eye from section shown in A, more highly magnified.

that leads to their abortion? In no instance was the abnormal growth of the nervous tissue seen to impinge seriously on any of the surrounding structures. All the specimens so far recovered have been so young that there was as yet no development of functioning neuron chains. One eannot therefore invoke disturbance of neural regulation as a factor in causing the death of embryos. 
The tendency glibly to ascribe any embryological defect to some sort of inhibiting influence has long tended to narrow our outlook on the causation of congenital defects in general. In the abnormalities here presented we are clearly dealing with local overgrowth of a specific type of tissue as the critical factor producing the anomaly. The clear implication of this situation is that we must scrutinize possible causative agents in a totally different category from those that might inhibit growth. It must make us broaden our perspective to include consideration of factors akin to those which must be involved in the causation of neoplasms. It is hoped that the provocative character of these findings and the problems they raise may direct enough attention to the field so that the material of this type which is undoubtedly available will receive more thoughtful attention.

\section{SUMMARY}

Evidence suggesting the possibility that local overgrowth of the neural plate in early stages of its development might be a factor in the genesis of myeloschisis and spina bifida was presented at the 1946 Anatomists' Meetings. During the past year extension of this study to cover the other parts of the central nervous system has brought to light several instances of disturbances in brain development in which local overgrowth appears to be an underlying factor. Photomicrographs are presented showing examples of such areas of abnormal growth from specimens in the Michigan collection. In these embryos, ranging from 7.5 to $30 \mathrm{~mm}$, local portions of the walls of the neural tube are richly plicated as if they had grown too large for the space in the head which they are destined to occupy. In some cases the redundant brain walls have caused the head to become grotesquely high-crowned; in others the overgrown brain protrudes as an encephalocoele. A preliminary survey of the Carnegie collection shows the availability there of similar cases with which the series can be extended. These findings suggest that a causative mech- 
anism entirely different from an "arrest of derelopment" may lie behind certain types of embryological disturbances. If this concept is borne out it would indicate the desirability of giving increased attention to growth accelerating factors as possible causative agents in abnormal growth. 